\title{
Clinical characteristic and management of descending necrotizing mediastinitis: a retrospective study, Dr. Soetomo Hospital, Surabaya
}

\author{
Dhihintia Jiwangga \\ Department of Thoracic and Cardiovascular, Dr. Soetomo Hospital Surabaya, Surabaya, Indonesia \\ Correspondence to: Dhihintia Jiwangga. Department of Thoracic and Cardiovascular, Dr. Soetomo Hospital Surabaya, Surabaya, Indonesia. \\ Email: dhihintiajiwangga@yahoo.com.
}

\begin{abstract}
Background: Descending necrotizing mediastinitis (DNM) is a mediastinal infection that is usually an extension or process of spreading an infection originating from the neck. The spread of infection occurs due to the influence of gravity and negative intrathoracic pressure.

Methods: We collect data from medical record patients who presented with DNM at Dr. Soetomo General Hospital, Surabaya, Indonesia, from January 2013 to December 2017. We collect characteristic data of each patient, diagnostic, operative management and outcome.

Results: We have 56 patients met inclusion criteria, There were 50 patients came to our hospital with preoperative septicemia. The source of infection that we found, odontogenic 30 cases (53.57\%), we did cervicotomy substernal drainage for 54 cases $(96.43 \%)$, sternotomy 1 (1.79\%) and right thoracotomy $1(1.79 \%)$. Our mortality is $14.29 \% / 5$ years.

Conclusions: Transcervical mediastinal drainage and adequate debridement is effective. Transthoracic mediastinal drainage was depended on each case. Thoracotomy if locus infection or pus accumulation on pleura. Sternotomy approach if locus infection is wide spreading to mediastinum, right or left pleura.
\end{abstract}

Keywords: Mediastinitis; descending necrotizing

Received: 31 July 2017; Accepted: 11 October 2018; Published: 14 December 2018.

doi: 10.21037 /jovs.2018.12.01

View this article at: http://dx.doi.org/10.21037/jovs.2018.12.01

\section{Introduction}

Mediastinitis is a severe inflammatory process in the mediastinum (1). This is caused by downward spreading of head and neck infections, especially from the mouth or pharynx (pharyngeal, peritonsillar, dental or odontogenic abscesses) through the mediastinum $(2,3)$. It has a high mortality rate (about $40 \%$ ) which is made worse by delayed diagnosis and therapy. The risk factors for descending necrotizing mediastinitis (DNM) some study mention about old age, and diabetes mellitus, malignancy, liver cirrhosis, and renal failure (4). Patients suffering from mediastinitis are admitted to hospitals in very critical conditions and generalized sepsis.

The mediastinum is a space in the middle part of the thoracic cavity that is bordered by the mediastinal parietal pleura and extends from the superior thoracic inlet to the inferior diaphragm. It is divided into three parts: the anterior, middle and posterior mediastinum (5).

The anterior mediastinum is bounded by the sternum; posterior by the pericardium and aorta; superior by the thoracic inlet; and inferior to the diaphragm. In the mediastinum there are lymph nodes, thymus, adipose tissue and large blood vessels. The middle mediastinum is located behind the anterior mediastinum. There are heart, ascending aortic, aortic arch superior vena cava (SVC) and inferior vena cava (5).

\section{Methods}

This is a retrospective study which describes the characteristic, 
Table 1 Characteristics

\begin{tabular}{lc}
\hline Characteristics & Value (\%) \\
\hline Number of cases & $56(100.0)$ \\
Sex (male) & $40(71.43)$ \\
Mean of age (years) & 43.73 \\
Diabetes mellitus & $10(17.86)$ \\
Mean ICU stay (days) & 3 \\
Mean hospital stay (days) & 11.64 \\
Preoperative septicemia & $50(89.29)$ \\
Mortality & $8(14.29)$ \\
\hline
\end{tabular}

Table 2 Source of infection

\begin{tabular}{lc}
\hline Source of infection & $\mathrm{N}(\%)$ \\
\hline Odontogenic & $30(53.57)$ \\
Esophageal & $1(1.79)$ \\
Head and neck & $25(44.64)$ \\
Tracheobronchial & 0 \\
\hline
\end{tabular}

Table 3 Surgical approach

\begin{tabular}{lc}
\hline Surgical approach & $\mathrm{N}(\%)$ \\
\hline Cervicotomy substernal drainage & $54(96.43)$ \\
Sternotomy & $1(1.79)$ \\
Left thoracotomy & 0 \\
Right thoracotomy & $1(1.79)$ \\
\hline
\end{tabular}

management, and outcome of DNM cases in our hospital. The study was conducted at Dr. Soetomo General Hospital Surabaya, Indonesia from January 2013 to December 2017. All patients with a diagnosis of DNM who arrived at the emergency department. The patient was followed until discharged from the hospital.

The inclusion criteria in this study were all patients who came to the hospital with a clinical manifestation of severe infection of the neck and oral cavity, which was associated with the mediastinal cavity causing mediastinitis. In these patients a chest X-ray and CT scan were carried out. These patient patients get antibiotic therapy and undergo surgery.

All data is taken from our hospital medical record. Management of DNM cases currently depends on clinical assessment and radiological findings for all cases. Debridement procedure is carried out from the cervical area to the mediastinum. Some cases use a sternotomy or thoracotomy approach which depends on radiological findings. Chest tube placement, either intrapleural or substernal for effective drainage. Sometimes additional infusion catheter at ICS 2 mid-clavicle was needed for continuous irrigation. The wound was left open or sutured situation for secondary healing.

We use the 2 nd generation of cephalosporin and metronidazole as perioperative antibiotics then it converted to definitive antibiotics after the results of bacterial culture are released. Postoperative chest X-ray is performed for evaluation after surgery. Re-exploration was performed if there was still accumulation of pus caused by inadequate drainage.

\section{Results}

We have 56 patients met inclusion criteria of this study which mean of age was 44. In Table 1, the average length of intensive care unit (ICU) is 3 days, with the longest period of treatment in the ICU was 6-day. The average total length of hospital stay for our patients is 12 days, the shortest was 2 days and the longest was 23 days. There were 40 subject males and 16 females. Diabetes mellitus which known from history of illness and blood sugar test was only positive for 10 subjects.

There were 50 patients came to our hospital with preoperative septicemia and treated with 2 nd generation of cephalosporin and metronidazole before their definitive antibiotics were confirmed. Total mortality of our study was 8 subjects (14.29\%).

On Table 2 show the source of infection that we found, odontogenic 30 cases $(53.57 \%)$, head and neck 25 cases (44.64\%) and 1 case of esophageal perforation (1.79\%). There were no case extension to posterior mediastinum, middle mediastinum and no case extension to pleural only. We did cervicotomy substernal drainage for 54 cases (96.43\%), sternotomy $1(1.79 \%)$ and right thoracotomy $1(1.79 \%)$ as describes on Table 3.

All of our procedures were followed with substernal drain 44 cases $(78.57 \%)$, and substernal and intrapleural drain 12 cases $(21.43 \%)$. There were no left pleural drainage only or right pleural drainage only (see Table 4).

Typical of down spreading infection were extension to 
Table 4 Drain insertion

\begin{tabular}{lc}
\hline Drain insertion & $\mathrm{N}(\%)$ \\
\hline Substernal drain & $44(78.57)$ \\
Right intrapleural & 0 \\
Left intrapleural & 0 \\
Substernal and intrapleural & $12(21.43)$ \\
\hline
\end{tabular}

Table 5 Down spreading type

\begin{tabular}{lc}
\hline Down spreading type & $\mathrm{N}(\%)$ \\
\hline Anterior mediastinum & $44(78.57)$ \\
Middle mediastinum & 0 \\
Posterior mediastinum & 0 \\
Pleura & 0 \\
Mediastinum and pleura & $12(21.43)$ \\
\hline
\end{tabular}

anterior mediastinum, 44 cases $(78.57 \%)$ and extension to both pleural and mediastinum 12 cases (21.43\%) (Table 5).

\section{Discussion}

DNM is an acute infection which cause of spreading infection from severe cervical infection. Infections originating in the mouth or neck can spread to the retropharyngeal space and will be a spread down towards the mediastinum. This spreading was easier with breathing and negative intrathoracic pressure. Patients with mediastinitis usually came to hospital with serious illness and can suddenly progress to sepsis and death (4).

The neck has three cavities, each of which is related to the mediastinal space. They are pretracheal, perivascular, and prevertebral so if an infection occurs in the neck area, it will be very easy for spreading to the mediastinum through the three neck spaces (Figure 1) (6).

Estrera et al. defined a diagnostic criterion for DNM (7). There are: (I) clinical manifestation of severe oropharyngeal infection; (II) radiologic features of mediastinitis on CT; (III) documentation of necrotizing mediastinal infection at surgery or on postmortem examination; and (IV) established relationship between oropharyngeal infection and development of DNM (3). We usually can recognize a cervical infection in all patients. There are some inflammatory sign obviously like red, warm, swell, or sometimes pus

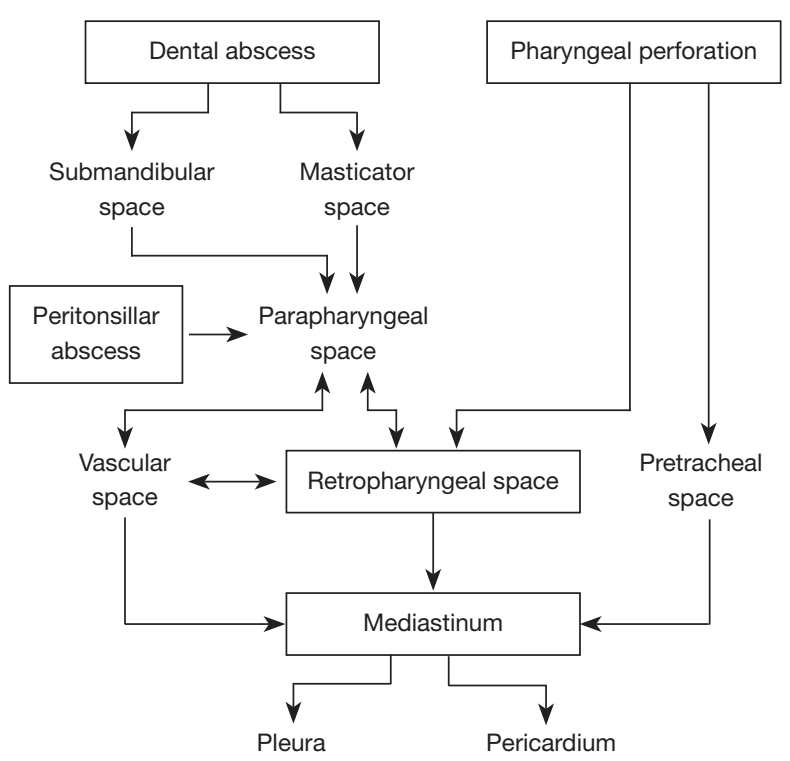

Figure 1 Pathways of oropharyngeal infections into the mediastinum (6).

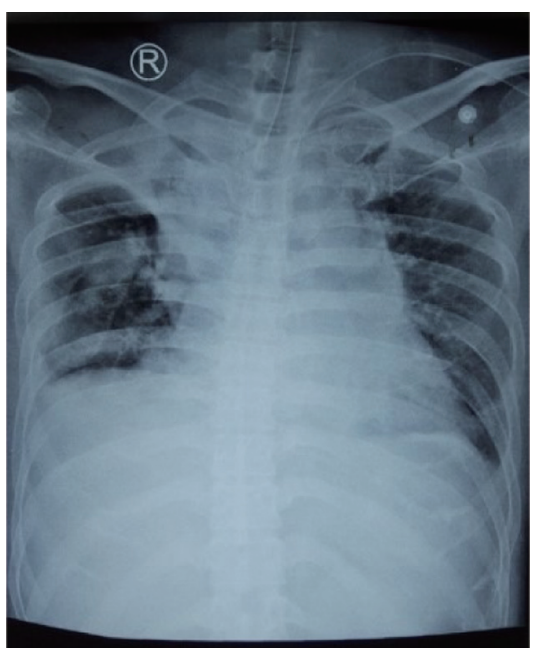

Figure 2 Widening mediastinum on chest $\mathrm{X}$-ray.

production made the diagnostic confirmed (8). Some patients came to hospital with seriously ill and septicemia sign. That was because the infection become serious and spread down to mediastinum. Chest $\mathrm{X}$-ray shows mediastinal widening in all patients (see Figure 2). It is recommended to do followup examination CT scan of the neck and chest cavity using contrast to confirm the diagnosis of necrotizing fasciitis in the neck extending to the mediastinum. We can see an 

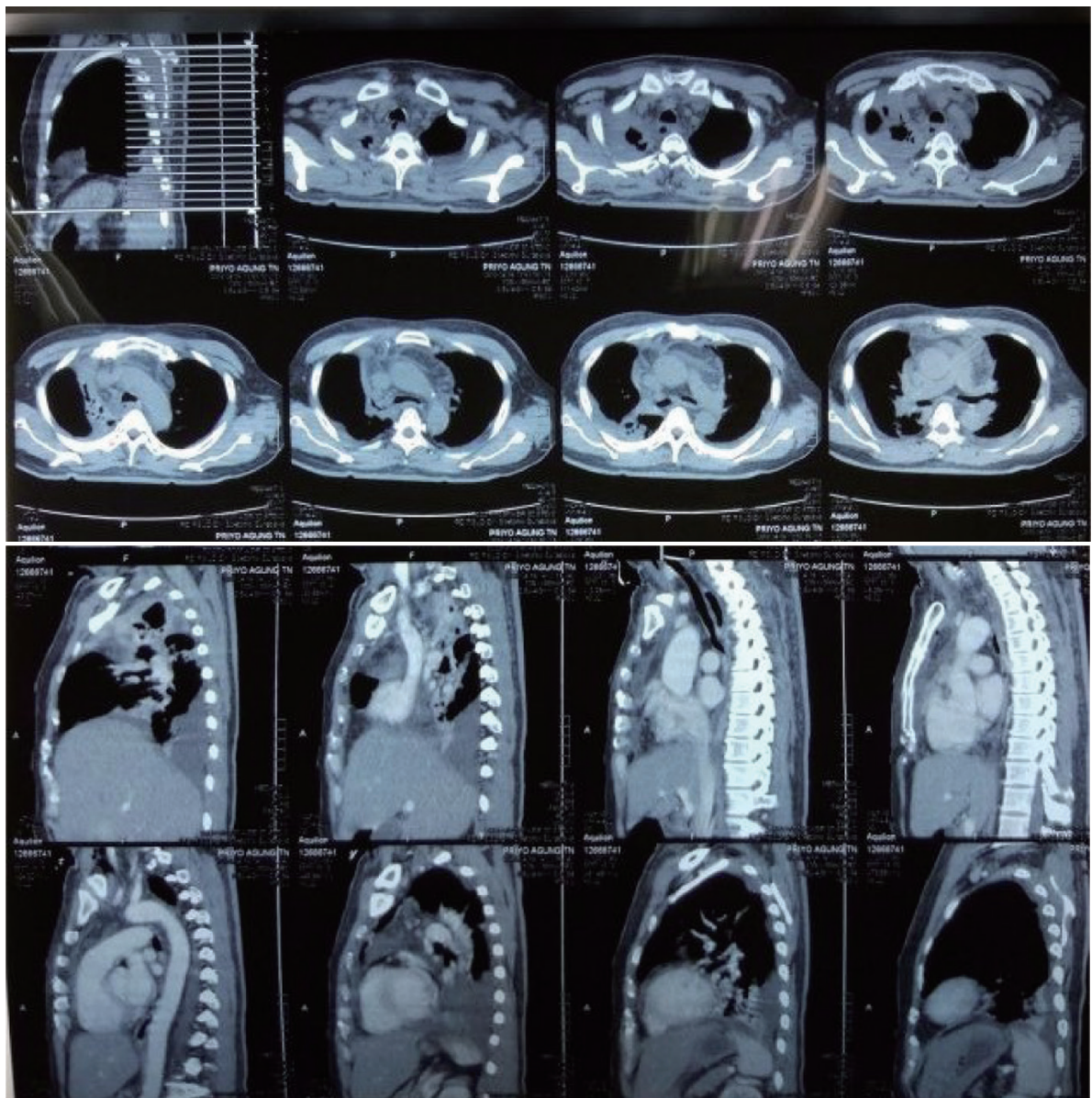

Figure 3 Chest CT showed pus accumulation on the neck and bilateral pleural effusion.

image of inflammation or accumulation of pus inside the neck that extends to the thoracic cavity (1) (see Figure 3).

The most common source of infection was from the teeth, followed by pharyngeal infection, primary neck infection, ingestion of foreign objects, or iatrogenic pharyngeal perforation due to instrumentation (9). According to our data showed in Table 2, the most of the potential emergencies that might arise from on odontogenic infection $(53.57 \%)$. Odontogenic infections can be divided into: dentoalveolar, periodontal, and facial plans. They can spread through contiguity causing jaws sinus, mandibular osteomielitis, cavernous sinus thrombophlebitis, internal jugular vein thrombophlebitis, erosion of the internal carotid artery, Ludwig's Angina and descending necrotic mediastinitis $(9,10)$. They can also spread in a hematogenic way causing an infective endocarditis (10). Whenever untreated, the infection will spread downwards into the mediastinum and cause a descending mediastinitis, a serious and fatal complication $(6,11)$. Down spreading type of infection mostly to the anterior mediastinum in our center (78.57\%). Sometimes spreading of infection goes through both mediastinum and pleura (21.43\%) (Table 5). There was no any subject had spreading type of infection pleura only, posterior mediastinum or middle.

\section{Medication}

Broad-spectrum intravenous antibiotics began to be given to all patients suspected of having cervical infection extending to the mediastinum. This antibiotics was given depend on our hospital microbial mapping which was later changed after the results of the germ culture taken from the pus specimen came out (12). We use the 2 nd generation of cephalosporin and metronidazole as perioperative antibiotics then it converted to definitive antibiotics after the results of bacterial culture are released. 


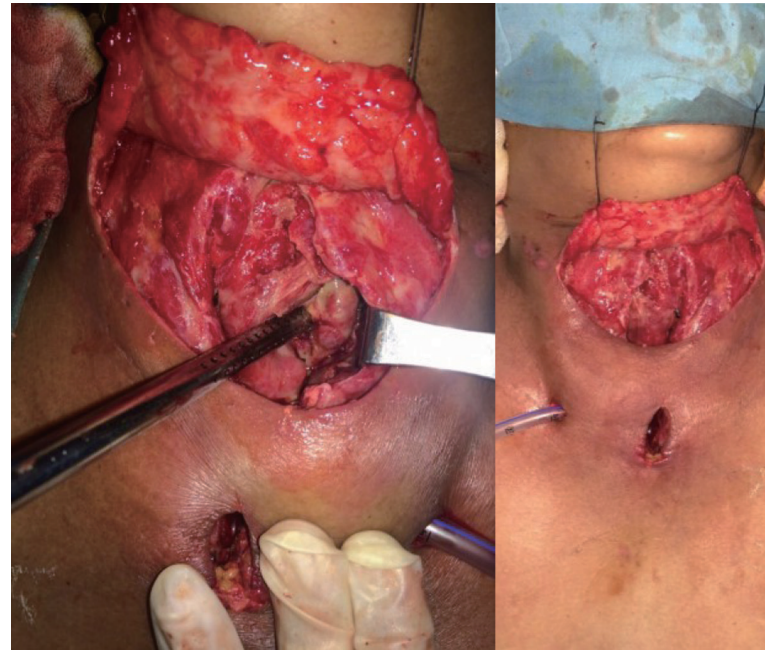

Figure 4 Adequate neck debridement and drainage.

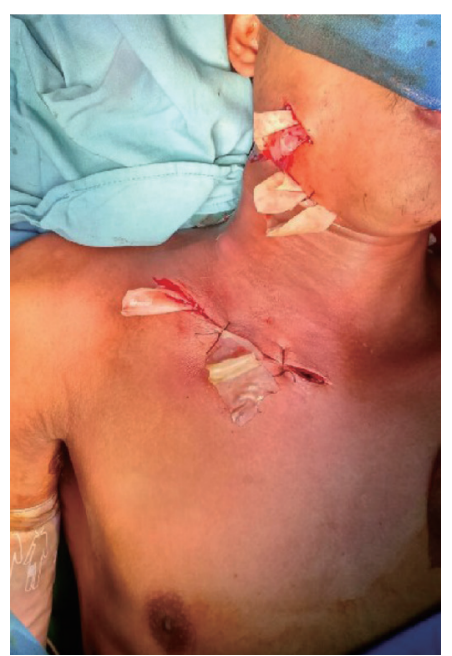

Figure 5 Pus accumulation was drainage and penrose drain inserted.

\section{Operative management}

There are some literatures describe several surgical approaches for adequate transthoracic drainage and debridement for DNM patients. There are median sternotomy, posterolateral thoracotomy, clam-shell, subxiphoid, and thoracoscopic. Some studies have reported their advantages and disadvantages for each technique $(6,13,14)$.

The surgical management for DNM is radically debriding any necrotic tissue present in the cervical region and the mediastinal space, including drainage from each abscess site and reducing airway obstruction. Debridement of cervical area usually via cervicotomy through an incision anterior sternocleidomastoid muscle on the involved side (s) or through a neck collar incision. The aims are open an involved cervical space, drainage (see Figure 4), and debrided of necrotic tissue (14). Transcervical mediastinal drainage and debridement procedures of the neck were done for 54 patients (Table 3). In transcervical mediastinal drainage, the superior mediastinum, the pretracheal, periesophageal and perivascular spaces were dissected manually through neck incisions $(6,14)$. Then, one or more soft tubes or Penrose drains were positioned in the open wounds in the upper mediastinum (6) (see Figure 5). One patient underwent transthoracic mediastinal drainage via a right thoracotomy approach. The right upper mediastinal pleura were opened longitudinally, and blunt dissection of the trachea, carina, periesophageal spaces, and perivascular spaces was performed. This procedure included radical debridement, decortication, and pleural drainage via chest tubes. Median sternotomy approach was used in one case because accumulation of pus in three chamber, mediastinum, right pleura and left pleura. The advantage of sternotomy is with a single incision, more optimal area for assessment, dilution, and drainage of the mediastinal compartment and both pleura (14). Unfortunately, the possibility of sternal dehiscence and sternal osteomyelitis made many surgeons think over from using this approach $(15,16)$.

Drain insertion was following surgery and pus location. All of our procedures were followed with substernal drain 44 cases $(78.57 \%)$, and substernal and intrapleural drain 12 cases $(21.43 \%)$. There were no left pleural drainage only or right pleural drainage only. The drainage tubes were removed when there was clinical improvement, serous and minimal production. We also used to check from chest X-ray before drain removal. We didn't ask chest CT evaluation due to cost effectiveness.

\section{Conclusions}

DNM is a critical infection of the mediastinal connective and it is a life threatening condition. The most cause of infection was odontogenic and usually it was spreading to anterior mediastinum. Transcervical mediastinal drainage and adequate debridement following with penrose drain and substernal drain is effective. Transthoracic mediastinal 
drainage was depended on each case. Thoracotomy if locus infection or pus accumulation on pleura. Sternotomy approach if locus infection is wide spreading to mediastinum, right or left pleura. Be careful of sternal wound dehiscence if we choose sternotomy approach.

\section{Acknowledgments}

Funding: None.

\section{Footnote}

Provenance and Peer Review: This article was commissioned by the Guest Editor (Kamran Ali) for the series "Asia Thoracoscopic Surgery Education Program (ATEP) Special Issue on Inflammatory Thoracic Diseases" published in Journal of Visualized Surgery. The article has undergone external peer review.

Conflicts of Interest: The author has completed the ICMJE uniform disclosure form (available at http:// dx.doi.org/10.21037/jovs.2018.12.01). The series "Asia Thoracoscopic Surgery Education Program (ATEP) Special Issue on Inflammatory Thoracic Diseases" was commissioned by the editorial office without any funding or sponsorship. The author has no other conflicts of interest to declare.

Etbical Statement: The author is accountable for all aspects of the work in ensuring that questions related to the accuracy or integrity of any part of the work are appropriately investigated and resolved. The study was conducted in accordance with the Declaration of Helsinki (as revised in 2013). The institutional ethics board approval was waived. Individual informed consent was waived due to the retrospective nature of the study.

Open Access Statement: This is an Open Access article distributed in accordance with the Creative Commons Attribution-NonCommercial-NoDerivs 4.0 International License (CC BY-NC-ND 4.0), which permits the noncommercial replication and distribution of the article with the strict proviso that no changes or edits are made and the original work is properly cited (including links to both the formal publication through the relevant DOI and the license). See: https://creativecommons.org/licenses/by-nc-nd/4.0/.

\section{References}

1. Abu-Omar Y, Kocher GJ, Bosco P, et al. European Association for Cardio-Thoracic Surgery expert consensus statement on the prevention and management of mediastinitis. Eur J Cardiothorac Surg 2017;51:10-29.

2. Gorospe Sarasúa L, Valdebenito-Montecino AP, MunozMolina GM. Descending Necrotizing Mediastinitis Secondary to Spontaneous Sternocleidomastoid Muscle Abscess. Arch Bronconeumol 2017;53:157-8.

3. Filiaci F, Riccardi E, Mitro V, et al. Disseminated necrotic mediastinitis spread from odontogenic abscess: our experience. Ann Stomatol (Roma) 2015;6:64-8.

4. Palma DM, Giuliano S, Cracchiolo AN, et al. Clinical features and outcome of patients with descending necrotizing mediastinitis: prospective analysis of 34 cases. Infection 2016;44:77-84.

5. Chauhan V, Thakur S. Extensive descending necrotizing mediastinitis can be managed conservatively. Int J Crit Illn Inj Sci 2015;5:219-20.

6. Buchholz BM, Kania A, Kaminski M. Descending necrotizing mediastinitis: Treatment concept with focus on thoracic surgery. Chirurg 2016;87:585-92.

7. Estrera AS, Landay MJ, Grisham JM, et al. Descending necrotizing mediastinitis. Surg Gynecol Obstet 1983;157:545-52.

8. Brotfain E, Koyfman L, Saidel-Odes L, et al. Deep Neck Infection and Descending Mediastinitis as a Complication of Propionibacterium acnes Odontogenic Infection. Case Rep Infect Dis 2015;2015:190134.

9. Cariati P, Monsalve-Iglesias F, Cabello-Serrano A, et al. Cervical necrotizing fasciitis and acute mediastinitis of odontogenic origin: A case series. J Clin Exp Dent 2017;9:e150-e152.

10. Ventura Spagnolo E, Mondello C, Cardia L, et al. Odontogenic abscess complicated by descending necrotizing mediastinitis: evidence of medical and dental malpractice. Minerva Stomatol 2016;65:412-5.

11. Mazzella A, Santagata M, Cecere A, et al. Descending necrotizing mediastinitis in the elderly patients. Open Med (Wars) 2016;11:449-60.

12. Ambrosch A. Rational antibiotic treatment of mediastinitis. Chirurg 2016;87:497-503.

13. Chen IC, Hsu JH, Dai ZK. Management of descending necrotizing mediastinitis complicated with empyema by video-assisted thoracoscopic surgery. Pediatr Pulmonol 
2016;51:E17-20.

14. Krüger M, Decker S, Schneider JP, et al. Surgical treatment of acute mediastinitis. Chirurg 2016;87:478-85.

15. Kaul P. Sternal reconstruction after post-sternotomy

doi: 10.21037/jovs.2018.12.01

Cite this article as: Jiwangga D. Clinical characteristic and management of descending necrotizing mediastinitis: a retrospective study, Dr. Soetomo Hospital, Surabaya. J Vis Surg 2018;4:246. mediastinitis. J Cardiothorac Surg 2017;12:94.

16. Ried M, Geis S, Potzger T, et al. Surgical reconstructive procedures of the chest wall after mediastinitis. Chirurg 2016;87:489-96. 